\title{
From misbelieve to proofs in transanal irrigation for functional bowel problems
}

\author{
P. Christensen ${ }^{1}$
}

Received: 15 July 2016/Accepted: 19 July 2016/Published online: 22 August 2016

(C) Springer-Verlag Italia Srl 2016

The Roman writer Pliny the Elder (AD 23-79) wrote in his encyclopedia about the knowledge of his time, how the ancient Egyptians took lessons from their sacred Ibis bird which at the banks of the Nile took the flood water with its curved beak and inserted the water into its anus to wash its intestines. References to the use of transanal irrigation can be found in the Papyrus Ebers (app. 1500 BC) and the Chester Beatty VI papyri (app. 1200 BC) [1].

Until the beginning of the nineteenth century, physicians only had few effective treatments and therefore took refuge in the 'concept of positive health,' and throughout the medical history, bowels have been flushed. With the discovery of bacteria as the origin of infectious diseases, use of bowel irrigation once again peaked. The concept of 'autointoxication' had developed in which bacteria and byproducts of incomplete digestion were thought to cross the barrier of the bowel wall and poison the body. The list of vague symptoms was long and included nausea, fatigue, depression, headache, anxiety, rheumatism, and constipation. Regular bowel movements became an obsession, and bowels were flushed intensively by the bourgeoisie. The theory of autointoxication became the ruling doctrine of medicine as the 'disease of diseases' [2]. However, in the established medical profession, faith in irrigation wavered as the effects of the treatment on the numerous diseases failed to materialize, while other pharmacological and surgical treatments revolutionized medicine.

P. Christensen

petchris@rm.dk

1 Pelvic Floor Unit, Department of Surgery P, Aarhus University Hospital, Tage Hansens gade 2, 8000 Aarhus C, Denmark
These misapprehensions form the backdrop of the present use of transanal irrigation as a treatment for constipation and fecal incontinence.

Scheduled irrigation may prevent fecal leakage between washouts and re-establish control over the time and place of defecation. Furthermore, regular evacuation of the rectosigmoid prevents constipation [3]. Transanal irrigation is rapidly gaining popularity in neurogenic bowel dysfunction for both pediatric and adult use $[4,5]$, in functional constipation and fecal incontinence [6], and in patients suffering from functional bowel problems after rectal surgery. Further, several commercial systems have been introduced to the medical device market. Use of transanal irrigation has been shown to reduce episodes of fecal incontinence, it is well tolerated by patients, and it improves quality of life. With imaging techniques, the effectiveness of emptying the left colon has been demonstrated [3] and anal physiology measures have been used in the search for predictors of successful treatment [7].

In patients with neurogenic bowel dysfunction, transanal irrigation also reduces time spent on bowel management, reduces the number of episodes of urinary tract infections, and has been shown to result in a lower total cost to society than standard bowel management. In a recent healthcare economic study, transanal irrigation was a cost-saving treatment strategy for patients with neurogenic bowel dysfunction who have failed standard bowel care for more than 6 months [8]. Moreover, the treatment improves quality-adjusted life expectancy (QALE) and reduces the number of episodes of fecal incontinence and urinary tract infections, as well as the risk of stoma surgery. Although the treatment is associated with considerable expense, there is a considerable cost saving to society overall and it was therefore recommended to be implemented as a standard treatment for neurogenic bowel dysfunction, when standard bowel care is insufficient. 
However, most of the evidence is based on retrospective single-center experiences, few prospective patient series and surveys such as the one from a Dutch tertiary referral center published in the current issue [6]. Randomized controlled trials outside the neurogenic bowel dysfunction group do not exist, and studies on reason for treatment failure are strongly needed. Clearly, transanal irrigation is not the solution for every patient with functional bowel problems. A significant proportion of patients dislike the treatment and will never start. There is an early dropout rate of $20-25 \%$ within the first weeks of treatment primarily due to lack of effect. Regarding long-term use, less than $50 \%$ of patients are on active treatment $[6,7]$. Furthermore, side effects are common and often refractory. Severe, life-threatening complications due to bowel perforation can occur. This is, however, very rare with an estimated risk of irrigation-induced perforation of less than 1 in 500,000 irrigation procedures [9]. Irrigation-induced bowel perforation is associated with prior pelvic surgery and is most common when treatment is initiated.

Successful treatment and safe use are, however, heavily dependent on careful patient selection, early patient motivation and respect of the absolute and relative contraindications. Dedicated healthcare professionals experienced in the field of transanal irrigation should be involved to instruct patients, to ensure proper handling of the catheter, and explain to them how to achieve the best results as well as provide information about side effects and the potential risk [10].

The complex pathophysiology leading to bowel dysfunction needs further study, and the mechanism of action of irrigation is still not fully understood. Is it a simple mechanical washout and gravity-led emptying of the diluted bowel contents, or is it stimulation of intrinsic neural reflexes? Which individual patient factors can contribute to successful treatment? The influence of the different components of the procedure (the frequency of irrigation, the volume of water used, the flow rate, timing, use of additional oral laxatives or additives to the irrigation fluid to support the effect) is not known. The type of catheter used might also influence outcome. When treatment is insufficient, how should it be adjusted in the individual patient to improve adherence to treatment? To guide future patient selection, well-designed controlled trials are needed. This is particularly important with regard to new indications such as low anterior resection syndrome.
Overall, transanal irrigation has established itself as a simple therapeutic method for management of fecal incontinence and constipation following failure of standard bowel care with functional improvement, improvement in quality of life and socialization, and avoidance of expensive neuromodulation and stoma surgery. However, more quality research is needed to strengthen the scientific proof of that irrigation that can serve as a simple treatment for functional bowel problems.

\section{Compliance with ethical standards}

Conflict of interest Peter Christensen has served as an external consultant and advisory board member for Coloplast and has served as an advisory board member for Wellspect Health Care.

\section{References}

1. Doyle D (2005) Per rectum: a history of enemata. J R Coll Phys Edinb 35:367-370

2. Chen TS, Chen PS (1989) Intestinal autointoxication: a medical leitmotif. J Clin Gastroenterol 11:434-441

3. Christensen P, Olsen N, Krogh K, Bacher T, Laurberg S (2003) Scintigraphic assessment of retrograde colonic washout in fecal incontinence and constipation. Dis Colon Rectum 46:68-76

4. Del Popolo G, Mosiello G, Pilati C et al (2008) Treatment of neurogenic bowel dysfunction using transanal irrigation: a multicenter Italian study. Spinal Cord 46:517-522

5. Christensen P, Bazzocchi G, Coggrave M et al (2006) A randomized, controlled trial of transanal irrigation versus conservative bowel management in spinal cord-injured patients. Gastroenterology 131:738-747

6. Vollebregt PF, Elfrink AKE, Meijerink WJHJ, Felt-Bersma RJF (2016) Results of long-term retrograde rectal cleansing in patients with constipation or fecal incontinence. Tech Coloproctol. doi:10. 1007/s10151-016-1502-y

7. Christensen P, Krogh K, Buntzen S, Payandeh F, Laurberg S (2009) Long-term outcome and safety of transanal irrigation for constipation and fecal incontinence. Dis Colon Rectum $52: 286-292$

8. Emmanuel A, Christensen P, Kumar G et al (2015) Long-term cost-effectiveness of transanal irrigation in patients with neurogenic bowel dysfunction who have failed standard bowel care. Value Health 18:A360

9. Christensen P, Krogh K, Perrouin-Verbe B et al (2016) Global audit on bowel perforations related to transanal irrigation. Tech Coloproctol 20:109-115

10. Emmanuel AV, Krogh K, Bazzocchi G, Members of working group on Trans Anal Irrigation from UK, Denmark, Italy, Germany, France and Netherlands et al (2013) Consensus review of best practice of transanal irrigation in adults. Spinal Cord $51: 732-738$ 